\title{
BMJ Open Risk factors for acute exacerbations of COPD in a primary care population: a retrospective observational cohort study
}

\author{
Hana Müllerová, ${ }^{1}$ Amit Shukla, ${ }^{1}$ Adam Hawkins, ${ }^{2}$ Jennifer Quint ${ }^{3}$
}

To cite: Müllerová $\mathrm{H}$, Shukla A, Hawkins A, et al. Risk factors for acute exacerbations of COPD in a primary care population: a retrospective observational cohort study. BMJ Open 2014;4:e006171. doi:10.1136/bmjopen-2014006171

- Prepublication history for this paper is available online. To view these files please visit the journal online (http://dx.doi.org/10.1136/ bmjopen-2014-006171).

Received 21 July 2014 Revised 17 October 2014 Accepted 28 November 2014

\section{(1) CrossMark}

${ }^{1}$ Worldwide Epidemiology, GlaxoSmithKline, Uxbridge, UK

${ }^{2}$ Global Respiratory

Franchise, GlaxoSmithKline, Uxbridge, UK

${ }^{3}$ London School of Hygiene and Tropical Medicine, London, UK

Correspondence to Dr Hana Müllerová; hana.x.muellerova@gsk.com

\section{ABSTRACT}

Objectives: To evaluate risk factors associated with exacerbation frequency in primary care. Information on exacerbations of chronic obstructive pulmonary disease (COPD) has mainly been generated by secondary carebased clinical cohorts.

Design: Retrospective observational cohort study. Setting: Electronic medical records database (England and Wales).

Participants: 58589 patients with COPD aged $\geq 40$ years with COPD diagnosis recorded between 1 April 2009 and 30 September 2012, and with at least 365 days of follow-up before and after the COPD diagnosis, were identified in the Clinical Practice Research Datalink. Mean age: 69 years; $47 \%$ female; mean forced expiratory volume in 1s $60 \%$ predicted.

Outcome measures: Data on moderate or severe exacerbation episodes defined by diagnosis and/or medication codes 12 months following cohort entry were retrieved, together with demographic and clinical characteristics. Associations between patient characteristics and odds of having none versus one, none versus frequent $(\geq 2)$ and one versus frequent exacerbations over 12 months follow-up were evaluated using multivariate logistic regression models.

Results: During follow-up, $23 \%$ of patients had evidence of frequent moderate-to-severe COPD exacerbations ( $24 \%$ one; $53 \%$ none). Independent predictors of increased odds of having exacerbations during the follow-up, either frequent episodes or one episode, included prior exacerbations, increasing dyspnoea score, increasing grade of airflow limitation, females and prior or current history of several comorbidities (eg, asthma, depression, anxiety, heart failure and cancer).

Conclusions: Primary care-managed patients with COPD at the highest risk of exacerbations can be identified by exploring medical history for the presence of prior exacerbations, greater COPD disease severity and co-occurrence of other medical conditions.

\section{INTRODUCTION}

Chronic obstructive pulmonary disease (COPD) is characterised by the progressive deterioration of lung function. In many patients, the course of the disease is

\section{Strengths and limitations of this study}

- We report the results of a retrospective cohort study of 58589 patients identified in the UK Clinical Practice Research Datalink (CPRD) with spirometry-confirmed chronic obstructive pulmonary disease (COPD) diagnosis during a 42-month period from 1 April 2009, with the primary objective of evaluating risk factors for acute exacerbations of COPD.

- Strengths of the study include: the large size of the cohort; the cohort is representative of the UK primary care population; the utilisation of the CPRD, a comprehensive and well-established electronic medical records database which incorporates spirometric scores and dyspnoea grades recorded as part of the Quality Outcomes Framework.

- The main limitation of this study is that the event recording of acute exacerbations in the CPRD is not entirely standardised, necessitating the use of an algorithm containing prescription records for oral corticosteroids and antibiotics as well as primary care visits for exacerbations, and accident and emergency department and hospital admissions for COPD to maximise the detection of exacerbations.

- Another possible study limitation is that the true population of patients with milder airflow obstruction and those who did not experience exacerbations may be under-represented in the data set due to such patients not seeking healthcare or undergoing spirometry.

punctuated by exacerbations, an acute worsening of symptoms which, in severe cases, can necessitate hospitalisation and even result in death. ${ }^{1}$ Exacerbations are the major cost driver, directly and indirectly, in COPD. ${ }^{2}$ COPD exacerbations are known to cluster in time and patients who have experienced one episode are at increased risk of a recurrent exacerbation. ${ }^{3}$ Furthermore, exacerbations accelerate the deterioration of lung function, ${ }^{4}$ which in turn increases the likelihood and severity of further exacerbations. 
Various definitions are used to mark the start, end, relapse and recurrence of exacerbations. ${ }^{5}{ }^{6}$ Frequent exacerbations, usually defined as two or more episodes per year, are the focus of the majority of clinical epidemiology studies, as they are considered a marker of greater disease burden and reduced survival. ${ }^{7}$ However, an increase in frequency of any moderate-to-severe exacerbation events has been associated with greater disease severity, and the best predictor of future events is a history of exacerbation $(\mathrm{s}) .^{7}$

Previous research into the natural history of COPD exacerbations has mainly focused on prospective cohorts of patients who have usually been recruited from secondary and/or tertiary care. Limited data are available on the burden of COPD exacerbations in patients who are managed in primary care. The use of electronic medical record (EMR) databases allows for the identification of very large-scale patient cohorts in a setting much more representative of clinical practice than prospective studies carried out in selective cohorts. EMR-based cohorts represent all patients diagnosed and treated in primary care, with information reflecting real-world healthcare provider decisions and patient behaviours without any interventions introduced. Furthermore, the retrospective cohort approach avoids the potential for behavioural biases associated with selection and active participation in research, the so-called 'Hawthorne effect'.

This retrospective, observational cohort study comprises patients who were identified from primary care using the UK Clinical Practice Research Datalink (CPRD-GOLD).

We aimed to evaluate records of episodes of moderate-to-severe exacerbations of COPD and to determine the factors associated with COPD exacerbation frequency in patients managed through primary care.

\section{METHODS}

\section{Study design}

We used a retrospective cohort study design and identified patients in the CPRD-GOLD who had a record of COPD diagnosis, defined as $\geq 1$ record of COPD-specific Read codes from 1 April 2009 until 30 September 2012. We then took the first record of COPD diagnosis during this predefined period and assigned it as a cohort entry date (cohort baseline). We then further limited the patient cohort to only those with a minimum of 365 days of available history in the database prior to and subsequent to this first record (figure 1).

\section{CPRD-GOLD database}

CPRD-GOLD, formerly known as the General Practice Research Database, is one of the largest computerised databases of linked anonymised primary care medical records in the world..$^{9}$ It reflects the complete EMR for all National Health Service (NHS) primary healthcare collected from approximately $8 \%$ of the population of England and Wales. ${ }^{10}$ All medical signs, symptoms,
Patients with $\geq 1$ record of COPD diagnosis after 1 April 2009: 107504

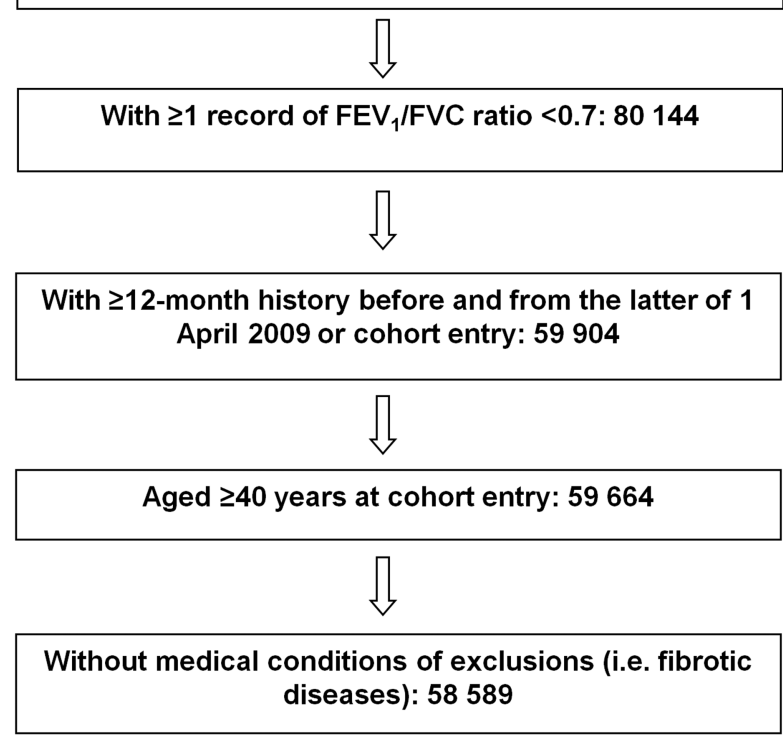

Figure 1 Patient recruitment flow chart. (COPD, chronic obstructive pulmonary disease; $\mathrm{FEV}_{1}$, forced expiratory volume in $1 \mathrm{~s}$; FVC, forced vital capacity).

investigations and diagnoses deemed important for the continued care of the patients are coded within the EMR. $^{10}{ }^{11}$

The CPRD research group, a part of the UK Medicines and Healthcare Products Regulatory Agency, continuously monitors data quality and removes practices from the database if they fail to maintain the required standards of data entry.

\section{Patient population}

The cohort consisted of patients with a record of COPD diagnosis between 1 April 2009 and 30 September 2012 who sought care for their condition; see Study design section. COPD diagnosis was required to be accompanied by spirometry recording consistent with obstructive disease, defined as a forced expiratory volume in $1 \mathrm{~s}$ $\left(\mathrm{FEV}_{1}\right)$ / forced vital capacity ratio of $<0.7$ recorded any time before and up to 3 months from the date of diagnosis of COPD record that qualified the patient to enter this cohort.

\section{Exacerbations of COPD}

An algorithm was assembled to collect data on recorded events of moderate-to-severe COPD exacerbations from the 12 months preceding and the 12 months subsequent to cohort entry date. Moderate exacerbations were defined as a record of a diagnosis of exacerbation, acute bronchitis, or the management of COPD with specific antibiotics and oral corticosteroids recorded on the same day or up to 5 days from each other. Admissions to hospital or visits to the accident and emergency department associated with COPD as recorded by general 
practitioners (GPs) were considered as severe exacerbations. An exacerbation episode was defined based on events recorded over 2 weeks from the initial exacerbation record. The start of an episode was considered to be the date of the first qualifying event. Subsequently, a 14-day rolling window was applied to identify a period of at least two exacerbation-free weeks in order to ensure that a relapse was not categorised as a separate exacerbation episode. ${ }^{12}$ The frequency of individual patients' exacerbations during the 12 months prior to the start of observation and also during the 12-month follow-up period was split into three categories: none, one, or two or more (frequent) episodes of moderate-to-severe exacerbation of COPD. The incidence of exacerbation episodes was also expressed as a rate per person per year, a sum of the episodes per patient divided by 365 days of follow-up. For a minority of patients, who would have been first diagnosed with COPD during the year prior to observation period start, we treated their exacerbation history similarly to those with established COPD.

\section{Other key study variables}

The following variables were also retrieved from the database using the latest record prior to cohort entry date: age, gender, smoking status, body mass index (BMI; or imputed BMI using the latest height and weight measurements where BMI data were not recorded), Medical Research Council (MRC) dyspnoea grade and $\mathrm{FEV}_{1}$ percent predicted. ${ }^{13}$

Mean BMI was summarised as both a continuous and categorical variable using the WHO classification of underweight $(<18.5)$, normal (18.5-24.9), overweight (25.0-29.9) and obese $(\geq 30){ }^{14}$

The GOLD grade classification ${ }^{15}$ was used to determine the severity of airflow limitation. Owing to the nature of the data available from CPRD-GOLD, it was not possible to determine whether the spirometry data had only been recorded following the administration of a bronchodilator. However, given that the NHS Quality Outcomes Framework requires all patients with COPD to have their COPD diagnosis confirmed with postbronchodilator spirometry, ${ }^{16}$ it is expected that the majority were recorded following bronchodilator administration. The categories of airflow severity limitation were defined using the $\mathrm{FEV}_{1}$ cut-off points $\geq 80 \%$ predicted for mild, $\geq 50 \%$ to $<80 \%$ predicted for moderate, $\geq 30 \%$ to $<50 \%$ predicted for severe and $<30 \%$ predicted for very severe airflow limitation.

MRC dyspnoea grade is a unidimensional measure of breathlessness related to activity. ${ }^{17}$ The MRC dyspnoea scale is equivalent to the modified MRC (mMRC) scale, which is an established method in the published literature. The key difference with the mMRC dyspnoea scale is a shift in the scoring range: whereas MRC ranged from 1 to 5, mMRC employs a range from 0 to 4 . Therefore, mMRC Grade 0 is equal to Grade 1 on the MRC scale. ${ }^{18}$
Records of diagnoses of selected comorbidities, including depression, anxiety and asthma, occurring prior to the start of the study were retrieved from the database. Data on the frequency of prescription treatment for COPD in the 12 months prior to the start of this cohort study were also collected. Patients who had one or more records for medication within a therapeutic class, with the exception of oral corticosteroids, were considered to use that medication. For oral corticosteroids, four or more prescriptions within the 12-month period were considered to indicate regular use rather than acute use for COPD exacerbation(s). Lastly, the number of recorded contacts with a GP for any reason in the 12 months before the cohort entry was ascertained and standardised per 365.25 days.

\section{Statistical analyses}

A multilevel multivariable logistic regression was used to assess risk factors associated with frequency of exacerbations over the 12-month follow-up period: frequent versus none, frequent versus one, and one versus none. These analyses were carried out using the PROC LOGISTICS procedures in SAS V.9.2. All covariates listed in descriptive tables, with the exception of respiratory medications, were entered into the models. Missing values for BMI and percent predicted $\mathrm{FEV}_{1}$ values (less than $2 \%$ of the cohort) and MRC dyspnoea score (12\% of the cohort) were not imputed.

The relationships between the number of exacerbation episodes during the 12-month follow-up period and the percent predicted $\mathrm{FEV}_{1}$ value and MRC dyspnoea grade were also assessed using Spearman's correlation coefficient.

\section{RESULTS \\ Patients}

The cohort consisted of 58589 patients with COPD (figure 1). Overall, $47 \%$ of patients were female, mean age was 69 years and mean $\mathrm{FEV}_{1}$ was $60 \%$ predicted.

\section{Incidence of exacerbation}

During the 12-month follow-up period, 53\% of patients ( $n=31049)$ had no recorded episodes of moderate-to-severe exacerbation of COPD, 24\% of patients $(n=14189)$ had one recorded exacerbation episode and frequent episodes were recorded in $23 \%$ of patients $(n=13351)$. The rate of moderate-to-severe exacerbations during the 12-month follow-up period was 0.89 per person per year. A maximum of eight episodes per patient was observed.

In comparison to patients with no recorded exacerbation episodes, patients with frequent exacerbations were more likely to be female ( $51 \%$ vs $44 \%$ ), had poorer lung function (mean $\mathrm{FEV}_{1}$ percent predicted: $54 \%$ vs $62 \%$ ) and more dyspnoea (MRC grade $\geq 3$ : $58 \%$ vs $39 \%$ ). When compared with patients with no recorded exacerbation episodes during the 12-month follow-up, patients with frequent exacerbations were more regularly 
diagnosed with comorbidities including myocardial infarction, heart failure and depression, and had been managed with maintenance treatment more often in the past 12 months ( $\geq 1$ prescribed inhaled corticosteroid/ long-acting $\beta$ agonist $68 \%$ vs $39 \%, \geq 1$ prescribed longacting muscarinic antagonist $53 \%$ vs $28 \%$; table 1 ).

Of the patients with no recorded exacerbation episodes during the 12-month follow-up period, $72 \%$ had no record of a moderate-to-severe exacerbation in the prior 12 months; $46 \%$ of patients who experienced frequent moderate-to-severe exacerbations during the follow-up period had also experienced two or more exacerbations in the prior 12 months (figure 2).

A weak, although statistically significant, relationship was observed between the frequency of COPD exacerbations and the stage of airflow limitation (Spearman's $\mathrm{r}=0.16, \mathrm{p}<0.001)$. Thirty-four per cent and $45 \%$ of patients with poor lung function (moderate and severe airflow limitation, respectively) also had no recorded exacerbations, in contrast to $16 \%$ and $19 \%$ of patients who had mild or moderate airflow limitation, respectively, with frequent exacerbation episodes (figure 3A).

\section{Factors associated with exacerbation frequency}

Comparison of patients with no exacerbation, one exacerbation and frequent exacerbations during the 12-month follow-up showed that several factors were associated with an increased likelihood of prospective frequent exacerbations; the strongest association was with prior exacerbations. Experiencing one moderate exacerbation, compared with none, during the 12 months preceding the start of the study was associated with one moderate-to-severe exacerbation occurring $(\mathrm{OR}=1.9)$ or frequent $(\geq 2)$ moderate-to-severe exacerbations (OR 3.3) occurring during the 12-month follow-up period (table 2). The OR for frequent $(\geq 2)$ moderate-to-severe exacerbations increased to 13.6 if patients had experienced two or more moderate episodes, compared with none in the 12 months preceding the start of the study.

The risk of frequent exacerbations compared with none, during the 12-month observation period, increased with greater severity of airway limitation; $\mathrm{OR}=1.2$ for moderate limitation $\left(\geq 50 \% \mathrm{FEV}_{1}<80 \%\right.$ predicted) and $\mathrm{OR}=2.4$ for very severe limitation $\left(\mathrm{FEV}_{1}>30 \%\right.$ predicted; table 2$)$. Increasing dyspnoea grade was also associated with an increased OR for frequent exacerbations compared with no exacerbations, from 1.4 for MRC2 to 3.0 for MRC5 when compared with MRC1 (table 2; figure 3B). The history of specific comorbidities was associated with a significant increase in the risk of patients experiencing frequent exacerbations compared with none during the observational period (table 2). Among the specific comorbidities assessed, history of asthma, cancer and depression had the strongest observed associations with exacerbation frequency, with corresponding ORs for frequent versus no exacerbations of 1.5, 1.3 and 1.3, respectively (table 2).
Similar risk factors for one versus no exacerbation, albeit of lower magnitude, were observed as for the risk of frequent versus no exacerbations.

\section{DISCUSSION}

We set out to explore COPD exacerbation frequency and associated factors, using a retrospective cohort approach, in a large primary care cohort of 58589 patients in England and Wales. We found marked differences between patients managed in primary care who had frequent exacerbations (23\% of patients) or one exacerbation (24\%) as compared with patients with no recorded COPD exacerbations (53\%) during the 12-month follow-up period.

The characteristic most strongly associated with moderate-to-severe exacerbation frequency was a history of exacerbations. A gradient relationship between moderate exacerbations in the prior 12 months and prospective moderate-to-severe exacerbations was apparent: patients who had no exacerbations in the prior 12 months had the lowest risk of future events; patients who had one exacerbation were at greater risk of future episodes than those who had none, but were at lower risk than those who had two or more prior exacerbations; patients with two or more prior exacerbations were at the highest risk of future exacerbations. Prior episodes of severe exacerbations also independently increased the odds of future exacerbations. This result is in agreement with previously reported findings. ${ }^{7}$ When moderate and severe prior exacerbations are considered separately, our findings provide additional supportive evidence of an independent and 'dose-like' relationship of prior moderate events and future exacerbation risk.

Exacerbation frequency also increased with increasing grade of airflow limitation and similarly with increasing dyspnoea score. A relationship between exacerbation frequency and symptom severity is well established on the basis of findings from prospective studies; ${ }^{19}{ }^{20}$ however, the two factors are semi-independent and patients with moderate airflow limitation can experience recurrent exacerbations. ${ }^{7}$ Again, airflow limitation and dyspnoea were associated with a higher risk for any exacerbation event, not only with frequent events. Likewise, the slightly increased probability of frequent exacerbations observed to be associated with females is consistent with previous reports. ${ }^{19}$ No relationships between age or smoking status and exacerbation frequency were observed.

Finally, we observed a relationship between a prior diagnosis of selected comorbidities in patient history and a risk of any future moderate-to-severe exacerbations. Consistent independent associations were observed between a history of asthma, depression, anxiety, heart failure and cancer and either one or two or more exacerbation episodes. It has previously been reported that COPD exacerbations are associated with cardiovascular events, including myocardial infarction 
Table 1 Characteristics of patients with COPD by frequency of recorded exacerbation episodes during 12-month follow-up

\begin{tabular}{|c|c|c|c|}
\hline & \multicolumn{3}{|c|}{ Moderate-to-severe exacerbation frequency } \\
\hline & None & One & Two or more \\
\hline Patient characteristic & $n=31049$ & $\mathrm{n}=14189$ & $n=13351$ \\
\hline Age, mean (SD) & $69.3(10.5)$ & $69.8(10.3)$ & $69.4(10.2)$ \\
\hline \multicolumn{4}{|l|}{ Smoking status } \\
\hline Current & $10531(34)$ & $4667(33)$ & $4173(31)$ \\
\hline Former & $15988(51)$ & $7474(53)$ & $7345(55)$ \\
\hline Never & $3312(11)$ & $1492(11)$ & $1301(10)$ \\
\hline Other & $1218(4)$ & $556(4)$ & $532(4)$ \\
\hline Female & $13619(44)$ & $6831(48)$ & $6832(51)$ \\
\hline BMI underweight $\left(<18.5 \mathrm{~kg} / \mathrm{m}^{2}\right)$ & $1327(4)$ & $641(5)$ & $751(6)$ \\
\hline BMI normal $\left(18.5-24.9 \mathrm{~kg} / \mathrm{m}^{2}\right)^{\prime}$ & $10533(34)$ & $4810(34)$ & $4668(35)$ \\
\hline BMI overweight $\left(25.0-29.9 \mathrm{~kg} / \mathrm{m}^{2}\right)$ & $10633(34)$ & $4778(34)$ & $4274(32)$ \\
\hline BMl obese $\left(\geq 30.0 \mathrm{~kg} / \mathrm{m}^{2}\right)$ & $8037(26)$ & $3760(26)$ & $3472(26)$ \\
\hline $\begin{array}{l}\text { Rate of moderate-to-severe exacerbations in prior } \\
12 \text { months/person/year, mean }\end{array}$ & 0.37 & 0.74 & 1.67 \\
\hline $\begin{array}{l}\text { Rate of exacerbations with hospital admission } \\
\text { in prior } 12 \text { months/person/year, mean }\end{array}$ & 0.07 & 0.12 & 0.2 \\
\hline \multicolumn{4}{|l|}{ Comorbidities any time in history } \\
\hline Acute myocardial infarction & $2530(8)$ & $1386(10)$ & $1350(10)$ \\
\hline Congestive heart disease & $1857(6)$ & $1041(7)$ & $1165(9)$ \\
\hline Depression & $3976(13)$ & $2116(15)$ & $2393(18)$ \\
\hline \multicolumn{4}{|l|}{$\geq 1$ prescription within 12 months before cohort entry } \\
\hline ICS & $5789(19)$ & $2883(20)$ & $2501(19)$ \\
\hline LABA & $2379(8)$ & $1278(9)$ & $1295(10)$ \\
\hline ICS/LABA & $12150(39)$ & $7328(52)$ & $9133(68)$ \\
\hline LAMA & $8618(28)$ & $5260(37)$ & 7049 (53) \\
\hline \multicolumn{4}{|l|}{ MRC dyspnoea scale score } \\
\hline MRC 1 & $6251(23)$ & $2059(16)$ & $1165(10)$ \\
\hline MRC 2 & $11552(42)$ & $4765(38)$ & $3546(31)$ \\
\hline MRC 3 & $6478(23)$ & $3487(28)$ & $3286(29)$ \\
\hline MRC 4 & 2959 (11) & $1876(15)$ & $2605(23)$ \\
\hline MRC 5 & $524(2)$ & $398(3)$ & $690(6)$ \\
\hline \multicolumn{4}{|l|}{ Airflow limitation grade } \\
\hline Mild & $5475(18)$ & $1971(14)$ & $1398(10)$ \\
\hline Moderate & $16815(54)$ & $7324(52)$ & $5693(43)$ \\
\hline Severe & $7013(23)$ & $3855(27)$ & 4629 (35) \\
\hline Very severe & $1152(4)$ & $814(6)$ & $1411(11)$ \\
\hline
\end{tabular}

All data are $\mathrm{n}(\%)$ unless otherwise stated. Percentages expressed as column $\%$.

BMI, body mass index; COPD, chronic obstructive pulmonary disease; ICS, inhaled corticosteroid; LABA, long-acting $\beta 2$-agonist; LAMA, long-acting muscarinic antagonist; MRC, Medical Research Council.

and heart failure, ${ }^{21}$ and the presence of these comorbidities increases the likelihood of mortality and costs associated with exacerbations. ${ }^{2}$ Likewise, the importance of comorbid asthma as a risk factor for more frequent exacerbation and accelerated disease progression has led to the description of an 'asthma-COPD overlap syndrome'. ${ }^{22}$ It has been estimated that overlap syndrome may be associated with up to a threefold increase in the frequency and severity of exacerbations. ${ }^{22}$ Overlap syndrome is typically seen in younger patients with a smoking burden that is below the average for patients with COPD, potentially providing a partial explanation of the absence of observed relationships between age, smoking status and exacerbation frequency in this study.

Analyses of this large, primary care-based cohort supported the established hypothesis of the existence of a 'frequent exacerbator' phenotype, widely reported in the COPD literature as an explanation for observations of a subgroup of patients who experience highly recurrent exacerbations. ${ }^{74}$ The pathophysiology underlying the frequent exacerbator phenotype is not yet fully understood, and identification of the inter-related factors is potentially of value in aiding the identification of patients who are likely to be at increased risk of further exacerbations. Early intervention is associated with faster recovery from exacerbation and improved health-related quality of life. ${ }^{25}$

The factors that we have observed to be associated with any future moderate-to-severe exacerbations are suggestive of a shared inflammatory biological mechanism that gives rise to further exacerbation events and also leads to a worsening of COPD symptoms. Markers 


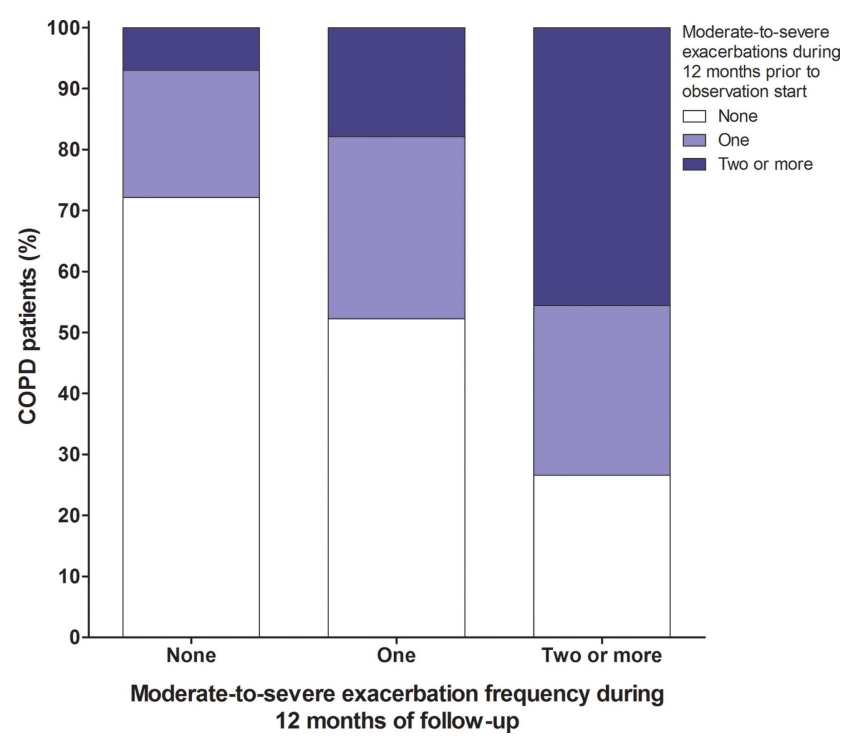

Figure 2 Frequency of moderate-to-severe chronic obstructive pulmonary disease (COPD) exacerbations in the 12 months prior to cohort entry in relation to the frequency of exacerbation episodes during the 12-month follow-up period.

for airway and systemic inflammation have been shown to be associated with exacerbation frequency, ${ }^{1}$ may precipitate lung function decline, ${ }^{19}$ and may also partly explain the observed relationships between exacerbation frequency and comorbidities such as cardiovascular disease $^{21} 26$ and asthma. ${ }^{27}$

We found that the characteristics of patients who experienced at least one exacerbation event during the follow-up period differed from those who did not have any exacerbations. This observation replicated earlier findings from a clinical cohort of 2138 patients enrolled in the Evaluation of COPD Longitudinally to Identify Predictive Surrogate Endpoints (ECLIPSE) study. ${ }^{7}$ However, it should also be noted that patients who do not experience exacerbations and have mild airflow limitation are less likely to seek healthcare and therefore less likely to be diagnosed with COPD. ${ }^{28}$

In this study, an inclusive definition of exacerbations was used, encompassing prescription records, as well as exacerbation event coding and GP records of hospital admissions and accident and emergency visits. Despite this, more than half of the cohort experienced no exacerbations during the 12-month observation period. It is possible that some exacerbations were unreported or self-managed at home with rescue packs of antibiotics and/or oral corticosteroids. Although a spirometry recording was required for eligibility, patients with COPD in the present study were identified based on a record of their diagnosis from a primary care database rather than actively recruited when visiting a physician. Therefore, we cannot ensure that all patients met the definition of COPD as per the GOLD guidelines. ${ }^{15}$ Further, we required all patients to be present in the database for at least a year postobservation period entry.
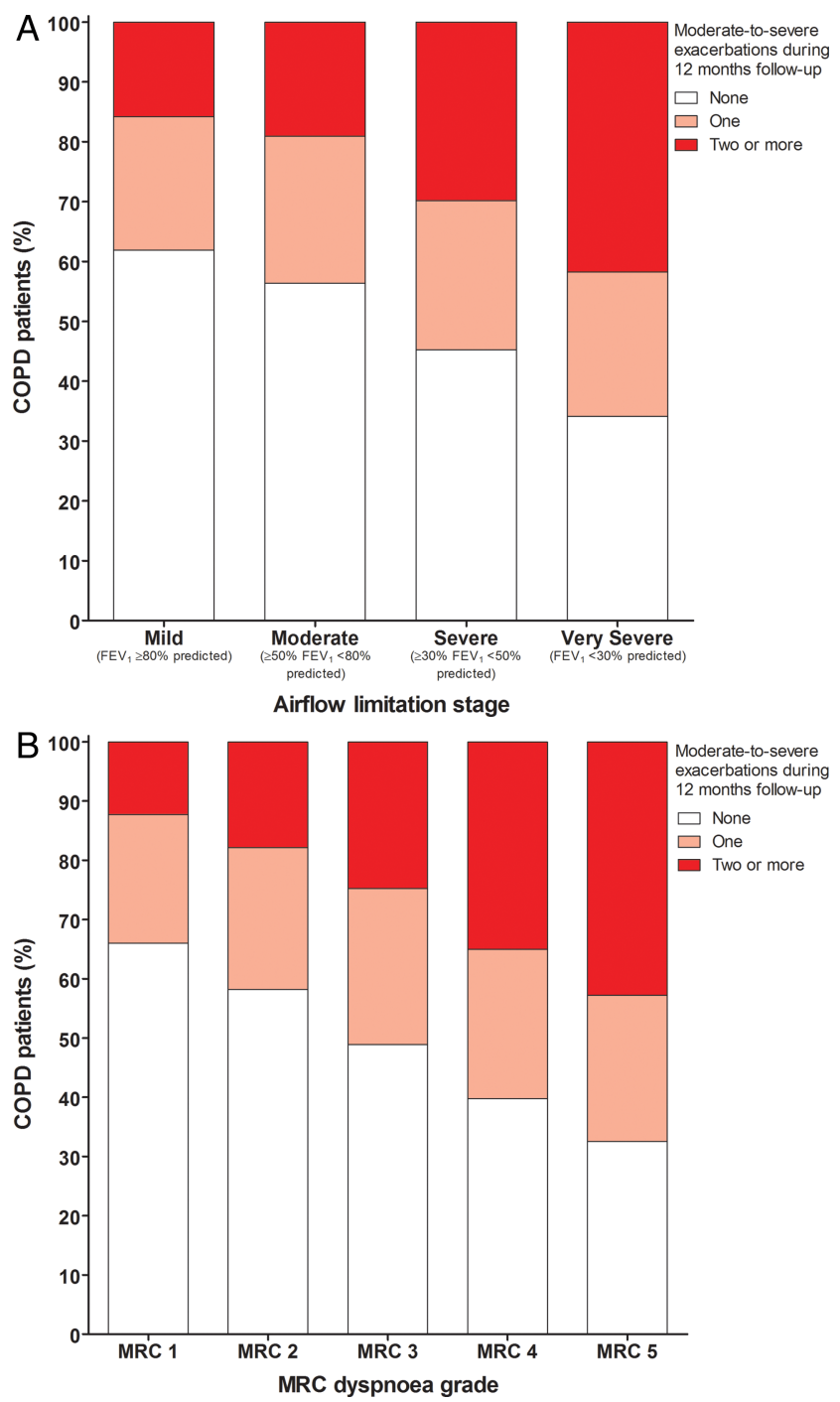

Figure 3 COPD exacerbation frequency during the 12-month follow-up period by $(A)$ airflow limitation stage and (B) MRC dyspnoea grade (COPD, chronic obstructive pulmonary disease; $F E V_{1}$, forced expiratory volume in $1 \mathrm{~s}$; MRC, Medical Research Council).

A fixed length of follow-up could have introduced a bias by removing the most severe patients from the analysis.

A strength of this study is the exploration of exacerbation data from a large sample of patients diagnosed with COPD obtained from a well-established, comprehensive primary care EMR-based data resource, the CPRDGOLD. This resource provides representative coverage of the patient population in England and Wales, and has incorporated spirometry and dyspnoea score data as a part of routine disease management of patients with COPD since 2004. CPRD-GOLD has previously been used in COPD research to determine disease prevalence in the $\mathrm{UK}^{29}$ and to investigate comorbidities in COPD and asthma. ${ }^{30} 31$

In conclusion, we demonstrated that, in a primary population of patients diagnosed with COPD, prior history of exacerbations (as well as increasing severity of airflow limitation and dyspnoea) and presence of 
Table 2 Factors associated with moderate-to-severe exacerbations during the 12-month follow-up period (three-category multilevel model)

\begin{tabular}{|c|c|c|c|c|c|c|}
\hline \multirow[b]{3}{*}{ Factor } & \multicolumn{6}{|c|}{ Moderate-to-severe exacerbations during 12-month follow-up } \\
\hline & \multicolumn{2}{|c|}{$\geq 2$ vs none } & \multicolumn{2}{|c|}{1 vs none } & \multicolumn{2}{|c|}{$\geq 2$ vs 1} \\
\hline & $\overline{\text { OR }}$ & $95 \% \mathrm{Cl}$ & $\overline{\text { OR }}$ & $95 \% \mathrm{Cl}$ & OR & $95 \% \mathrm{Cl}$ \\
\hline \multicolumn{7}{|c|}{ COPD exacerbations, 12 months prior to observation period start } \\
\hline No moderate event & \multicolumn{6}{|c|}{ Reference } \\
\hline 1 moderate episode & 3.31 & 3.12 to 3.51 & 1.89 & 1.79 to 1.99 & 1.76 & 1.65 to 1.87 \\
\hline$\geq 2$ moderate episodes & 13.64 & 12.67 to 14.68 & 3.11 & 2.88 to 3.37 & 4.41 & 4.11 to 4.74 \\
\hline No hospitalised episode & \multicolumn{6}{|c|}{ Reference } \\
\hline$\geq 1$ hospitalised episode & 2.13 & 1.95 to 2.31 & 1.49 & 1.38 to 1.61 & 1.44 & 1.32 to 1.57 \\
\hline \multicolumn{7}{|c|}{ Airflow limitation level nearest to observation period start } \\
\hline Mild $\left(\mathrm{FEV}_{1} \geq 80 \%\right.$ predicted) & \multicolumn{6}{|c|}{ Reference } \\
\hline Moderate $\left(\geq 50 \% \mathrm{FEV}_{1}<80 \%\right.$ predicted $)$ & 1.23 & 1.13 to 1.33 & 1.19 & 1.12 to 1.27 & 1.04 & 0.95 to 1.14 \\
\hline Severe $\left(\geq 30 \% \mathrm{FEV}_{1}<50 \%\right.$ predicted $)$ & 1.81 & 1.66 to 1.98 & 1.36 & 1.27 to 1.47 & 1.37 & 1.24 to 1.50 \\
\hline Very severe $\left(\mathrm{FEV}_{1}>30 \%\right.$ predicted $)$ & 2.37 & 2.08 to 2.70 & 1.59 & 1.41 to 1.79 & 1.56 & 1.37 to 1.78 \\
\hline \multicolumn{7}{|c|}{ MRC dyspnoea scale nearest to observation period start } \\
\hline 1 & \multicolumn{6}{|c|}{ Reference } \\
\hline 2 & 1.39 & 1.28 to 1.51 & 1.16 & 1.09 to 1.23 & 1.16 & 1.06 to 1.27 \\
\hline 3 & 1.86 & 1.70 to 2.03 & 1.41 & 1.31 to 1.51 & 1.32 & 1.20 to 1.44 \\
\hline 4 & 2.74 & 2.49 to 3.03 & 1.55 & 1.43 to 1.69 & 1.74 & 1.57 to 1.93 \\
\hline 5 & 3.02 & 2.58 to 3.55 & 1.62 & 1.39 to 1.88 & 1.84 & 1.57 to 2.16 \\
\hline \multicolumn{7}{|c|}{ Comorbidities, history of medical diagnosis before observation period start (absence is referent category) } \\
\hline Heart failure & 1.2 & 1.08 to 1.32 & 1.1 & 1.00 to 1.20 & 1.11 & 1.00 to 1.23 \\
\hline Myocardial infarction & 1.13 & 1.03 to 1.24 & 1.15 & 1.06 to 1.24 & 0.96 & 0.87 to 1.05 \\
\hline Rheumatological disease & 1.12 & 1.01 to 1.24 & 1.1 & 1.01 to 1.21 & 1.04 & 0.94 to 1.16 \\
\hline Renal disease & 0.9 & 0.84 to 0.97 & 0.96 & 0.91 to 1.02 & 0.93 & 0.86 to 1.00 \\
\hline Anxiety & 1.16 & 1.08 to 1.25 & 1.14 & 1.08 to 1.22 & 1.02 & 0.95 to 1.09 \\
\hline Depression & 1.25 & 1.16 to 1.35 & 1.1 & 1.02 to 1.17 & 1.12 & 1.04 to 1.21 \\
\hline Asthma & 1.51 & 1.43 to 1.60 & 1.24 & 1.19 to 1.30 & 1.23 & 1.16 to 1.30 \\
\hline Cancer & 1.28 & 1.19 to 1.38 & 1.1 & 1.03 to 1.17 & 1.14 & 1.05 to 1.23 \\
\hline \multicolumn{7}{|c|}{ Number of contacts with GP, 12 months prior to observation period start } \\
\hline Low: 0-5 & \multicolumn{6}{|c|}{ Reference } \\
\hline Medium: 6-9 & 1.02 & 0.95 to 1.10 & 1.07 & 1.00 to 1.13 & 0.99 & 0.91 to 1.07 \\
\hline High: $\geq 10$ & 1.24 & 1.16 to 1.32 & 1.18 & 1.12 to 1.24 & 1.08 & 1.01 to 1.16 \\
\hline \multicolumn{7}{|l|}{ Sex } \\
\hline Female & 1.19 & 1.13 to 1.26 & 1.12 & 1.07 to 1.17 & 1.07 & 1.01 to 1.14 \\
\hline
\end{tabular}

Models further adjusted for age, smoking and BMI.

$\mathrm{BMI}$, body mass index; COPD, chronic obstructive pulmonary disease; $\mathrm{FEV}_{1}$, forced expiratory volume in $1 \mathrm{~s}$; GP, general practitioner; MRC, Medical Research Council.

comorbidities predict risk of future moderate-to-severe exacerbation events. We also showed that patients with prior frequent exacerbations, as well as those with only one prior moderate exacerbation, are at increased risk of future exacerbation events. As exacerbations can accelerate the progression of the disease, our findings underline the importance of identifying patients with COPD who are at risk of exacerbation and intervening as early as possible. Such identification is feasible for a primary care practitioner based on a review of clinical history routinely recorded in the UK electronic health record.

Collaborators Editorial support in the form of development of the manuscript first draft in consultation with the authors, editorial suggestions to draft versions of this paper, assembling tables and figures, collating author comments, copyediting, fact checking, referencing and graphic services was provided by lan Grieve, PhD, at Gardiner-Caldwell Communications, an Ashfield company (Macclesfield, UK).
Contributors HM wrote the study protocol and analysis plan, oversaw the data analysis and drafted the manuscript. JQ and $A H$ contributed to the conception and design of the study and the interpretation of the data. AS contributed to the analysis and the interpretation of the data.

Funding This study was funded by GSK R\&D. The funder provided support in the form of salaries for authors HM, AS and AH. The decision to submit for publication is required by GSK policy of publicly disclosing research activities.

Competing interests $\mathrm{HM}, \mathrm{AS}$ and $\mathrm{AH}$ are employees of and hold stock in GSK. JQ received funding from an MRC Population Health Scientist Fellowship, grant number G0902135, and declares no competing interests.

Ethics approval The study protocol (WEUSKOP5903) was reviewed and approved as Protocol 12_118 by the CPRD Scientific and Ethics committee.

Provenance and peer review The study protocol was peer-reviewed by the GSK R\&D Epidemiology Protocol Review Forum and the report was also peer-reviewed by the medical and epidemiology supervisors.

Data sharing statement Access to the data used in this analysis, CPRD-GOLD database, is governed by the Ethics and Scientific committee: http://www.cprd.com/isac/. The authors cannot allow access to the CPRD-GOLD database because of contractual and ethics regulations imposed 
by the CPRD and its Ethics committee. Additional statistical analyses included in the final report are available from the corresponding author.

Open Access This is an Open Access article distributed in accordance with the Creative Commons Attribution Non Commercial (CC BY-NC 4.0) license, which permits others to distribute, remix, adapt, build upon this work noncommercially, and license their derivative works on different terms, provided the original work is properly cited and the use is non-commercial. See: http:// creativecommons.org/licenses/by-nc/4.0/

\section{REFERENCES}

1. Donaldson GC, Seemungal TA, Patel IS, et al. Airway and systemic inflammation and decline in lung function in patients with COPD. Chests 2005;128:1995-2004.

2. Perera PN, Armstrong EP, Sherrill DL, et al. Acute exacerbations of COPD in the United States: inpatient burden and predictors of costs and mortality. COPD 2012;9:131-41.

3. Hurst JR, Donaldson GR, Quint JK, et al. Temporal clustering of exacerbations in chronic obstructive pulmonary disease. $A m \mathrm{~J}$ Respir Crit Care Med 2009;179:369-74.

4. Vestbo J, Edwards LD, Scanlon PD, et al. Changes in forced expiratory volume in 1 second over time in COPD. New Engl J Med 2011;365:1184-92.

5. Aaron SD, Donaldson GC, Whitmore GA et al. Time course and pattern of COPD exacerbation onset. Thorax 2012;67:238-43.

6. Leidy NK, Murray LT. Patient-reported outcome (PRO) measures for clinical trials of COPD: the EXACT and E-RS. COPD 2013;10:393-8.

7. Hurst JR, Vestbo J, Anzueto A, et al. Susceptibility to exacerbation in chronic obstructive pulmonary disease. New Engl J Med 2010;363:1128-38.

8. McCambridge J, Witton J, Elbourne DR. Systematic review of the Hawthorne effect: new concepts are needed to study research participation effects. J Clin Epidemiol 2014;67:267-77.

9. Tate AR, Beloff N, Al-Radwan B, et al. Exploiting the potential of large databases of electronic health records for research using rapid search algorithms and an intuitive query interface. J Am Med Inform Assoc 2014;21:292-8.

10. Williams JEA, Green $\mathrm{RH}$, Warrington V, et al. Development of the i-BODE: validation of the incremental shuttle walking test within the BODE index. Respir Med 2012;106:390-6.

11. Wood L, Martinez C. The general practice research database: role in pharmacovigilance. Drug Safety 2004;27:871-81.

12. Leidy NK, Wilcox TK, Jones PW, et al. Standardizing measurement of chronic obstructive pulmonary disease exacerbations. Reliability and validity of a patient-reported diary. American J Respir Crit Care Med 2011;183:323-9.

13. Bhaskaran K, Forbes HJ, Douglas I, et al. Representativeness and optimal use of body mass index (BMI) in the UK Clinical Practice Research Datalink (CPRD). BMJ Open 2013;3:e003389.

14. World Health Organisation. Obesity: preventing and managing the global epidemic. Report of a WHO Consultation. WHO Technical
Report Series 894. Geneva: World Health Organisation, 2000. http:// whqlibdoc.who.int/trs/WHO_TRS_894.pdf (accessed Jul 2014).

15. Global Initiative for Chronic Obstructive Lung Disease (GOLD). Global Strategy for the Diagnosis, Management and Prevention of Chronic Obstructive Pulmonary Disease. Revised 2014. http://www. goldcopd.org/uploads/users/files/GOLD_Report_2014_Jun11.pdf (accessed Jul 2014).

16. NICE. Quality and Outcomes Framework for COPD, NM63 Advisory Committee Recommendations. 2012. http://www.nice.org.uk/aboutnice/ qof/indicators detail.jsp?summary=13808 (accessed Jul 2014).

17. Fletcher CM, Elmes PC, Fairbairn AS, et al. The significance of respiratory symptoms and the diagnosis of chronic bronchitis in a working population. BMJ 1959;2:257-66.

18. Williams T, van Staa T, Puri S, et al. Recent advances in the utility and use of the General Practice Research Database as an example of a UK Primary Care Data resource. Ther Adv Drug Saf 2012;3:89-99.

19. Burge S, Wedzicha JA. COPD exacerbations: definitions and classifications. Eur Respir J 2003;21(Suppl. 41):46s-53s.

20. Donaldson GC, Wedzicha JA. COPD exacerbations. 1: epidemiology. Thorax 2006;61:164-8.

21. Donaldson GC, Hurst JR, Smith CJ, et al. Increased risk of myocardial infarction and stroke following exacerbation of COPD. Chest 2010;137:1091-7.

22. Hardin M, Silverman EK, Barr RG, et al. The clinical features of the overlap between COPD and asthma. Respir Res 2011:12:127.

23. De Marco R, Pesce G, Marcon A, et al. The coexistence of asthma and chronic obstructive pulmonary disease (COPD): prevalence and risk factors in young, middle-aged and elderly people from the general population. PLoS One 2013;8:e62985.

24. Wedzicha JA, Brill SE, Allinson JP, et al. Mechanisms and impact of the frequent exacerbator phenotype in chronic obstructive pulmonary disease. BMC Med 2013;11:181.

25. Wilkinson TMA, Donaldson GC, Hurst JR, et al. Early therapy improves outcomes of exacerbations of chronic obstructive pulmonary disease. Am J Respir Crit Care Med 2004;169:1298-303.

26. Halpin DMG, Decramer M, Celli B, et al. Risk of nonlower respiratory serious adverse events following exacerbations in the 4-year UPLIFT trial. Lung 2011;189:261-8.

27. Zeki AA, Schivo M, Chan A, et al. The asthma-COPD overlap syndrome: a common clinical problem in the elderly. J Allergy 2011:861926.

28. Bednarek M, Maciejewski J, Wozniak M, et al. Prevalence, severity and underdiagnosis of COPD in the primary care setting. Thorax 2008;63:402-7.

29. Soriano JB, Maier WC, Egger $\mathrm{P}$, et al. Recent trends in physician diagnosed COPD in women and men in the UK. Thorax 2000;55:789-94.

30. Soriano JB, Visick GT, Muellerova $\mathrm{H}$, et al. Patterns of co-morbidities in newly diagnosed COPD and asthma in primary care. Chest 2005;128:2099-107.

31. García Rodríguez LA, Wallander M-A, Martín-Merino E, et al. Heart failure, myocardial infarction, lung cancer and death in COPD patients: a UK primary care study. Respir Med 2010;104:1691-9. 